\title{
A RBF Neural Network Approach Towards Precision Motion System with Selective Sensor Fusion
}

\author{
Rui Yang ${ }^{\mathrm{a}}$, Poi Voon Er ${ }^{\mathrm{b}}$, Zidong Wang, ${ }^{\mathrm{a}, \mathrm{c}}$, Kok Kiong Tan ${ }^{\mathrm{b}}$ \\ ${ }^{a}$ College of Electrical Engineering and Automation, Shandong University of Science and Technology, Qingdao, China 266590 \\ ${ }^{b}$ Department of Electrical and Computer Engineering, National University of Singapore, Singapore 117583 \\ ${ }^{c}$ Department of Computer Science, Brunel University London, Uxbridge, Middlesex, UB8 3PH, United Kingdom
}

\begin{abstract}
A radial basis function (RBF) neural network approach with a fusion of multiple signal candidates in precision motion control is studied in this paper. Sensor weightages are assigned to sensor measurements according to the selector attributes and are approximated using RBF neural network in multi-sensor fusion. A specific application towards precision motion control of a linear motor system using a magnetic encoder and a soft position sensor in conjunction with an analog velocity sensor is demonstrated. Motion velocity and noise level in the sensor are chosen as the selector attributes and the optimal sensor weightages under different attributes are approximated using RBF neural network with the reference data from laser interferometer. The experiment results illustrate that the proposed method can provide relative better results than both single encoder measurement and ordinary RBF neural network based multi-sensor approach.
\end{abstract}

Keywords: Multiple sensor, RBF neural network, Position measurement, Precision motion system

\section{Introduction}

High precision motion control is a core requirement in many industries leveraging on precise robotics and automation such as semiconductor manufacturing, precision machining and metrology. The measurement accuracy sustainable in those applications relies critically on the precision and resolution of the position sensors used as well as other important characteristics such as response time, bandwidth, robustness to environmental factors, control interface, physical dimensions, mounting and price. Because there is inevitably a limit to the overall performance achievable with a single sensor, multiple sensors can be used and fused when multiple facets of performance measures are necessary in an application.

The approach using multi-sensor fusion to improve system performance has been used in certain domains, including location tracking systems [1], reverse engineering in coordinate measuring machines [2][3], terrain mapping [4], vehicle navigation [5] and robotics control [6]. In previous mentioned research, many probability based multi-sensor fusion techniques based on probabilistic modelling

Preprint submitted to Neurocomputing have been proposed, such as Bayes' rule, probabilistic grids, Kalman filter, sequential monte carlo methods and functional density estimates. However, due to the limitations in probabilistic methods such as unguaranteed precision in probabilities for the states of interest and inefficient implementation of large number of probabilities [7], the applications of multi-sensor fusion are relatively scarce in precision motion control.

Alongside the probability based methods, neural network method has been adopted by several researchers in the areas of multi-sensor data fusion, such as robot inverse kinematics determination [8] and tool condition monitoring [9]. In all the proposed neural network based data fusion methods from literature review, the measured data from multiple sensors are directly used as the inputs to train the network without any amenable structure to deal with the changes in the system attributes and the environment which may affect the output of each sensor. However, neural network based multisensor fusion may produce worse results than single sensor due to the lack of secondary information in the fusion [10], which lacks the flexibility and has weakness in uncertainty [11]. 
In order to overcome the previous mentioned limitations in sensor fusion techniques and to expand applications of such fusion techniques to precision motion system, a novel radial basis function (RBF) neural network based sensor fusion framework is proposed. The weight attached to each sensor measurement is not fixed but is allowed to evolve to selector attributes chosen to bring out the relative strengths of each measurement. As these weights change in a nonlinear manner to the selectors, a $\mathrm{RBF}$ network is used to model the weight variations over the range of operations under different selector attributes, which has a fast convergence rate and high accuracy due to the local approximation ability [8]. RBF method also has several advantages over the look-up table, such as parametric model, nonlinear interpolation, and recursively refined parameters. It has been shown that under mild assumptions, RBF is suitable for universal approximations, i.e., any continuous function can be approximated over a compact set to any degree of accuracy.

The proposed method is more efficient because processing selector attributes instead of sensor measurements in neural network is relatively faster and easier due to low data dimensions. The proposed method also provides higher flexibility to cope with uncertainties in secondary information. The difference between the proposed method and conventional neural network based sensor fusion method is illustrated in Fig. 1. In this paper, the approach is more specifically elaborated with respect to precision motion system where the application of sensor fusion is less commonly encountered and a case study is employed in the development which involves the motion control of a linear motor using a digital magnetic encoder and an analog circuit providing incremental position measurements from velocity measured by an analog sensor. In this way, this paper suggests a new approach to apply multisensor fusion in high precision system instead of conventional probability based method.

The essential difficulties encountered in this research are the weightage adaptation in RBF neural network training and the consistency in system measurement. In order to avoid the over-fitting and local minimal problems, the parameters such as training rates are properly selected in RBF neural network training and each network is trained multiple times to ensure it converges to minimum. Consistent measurement is also a critical requirement, as the accuracy of fusion outcome depends on

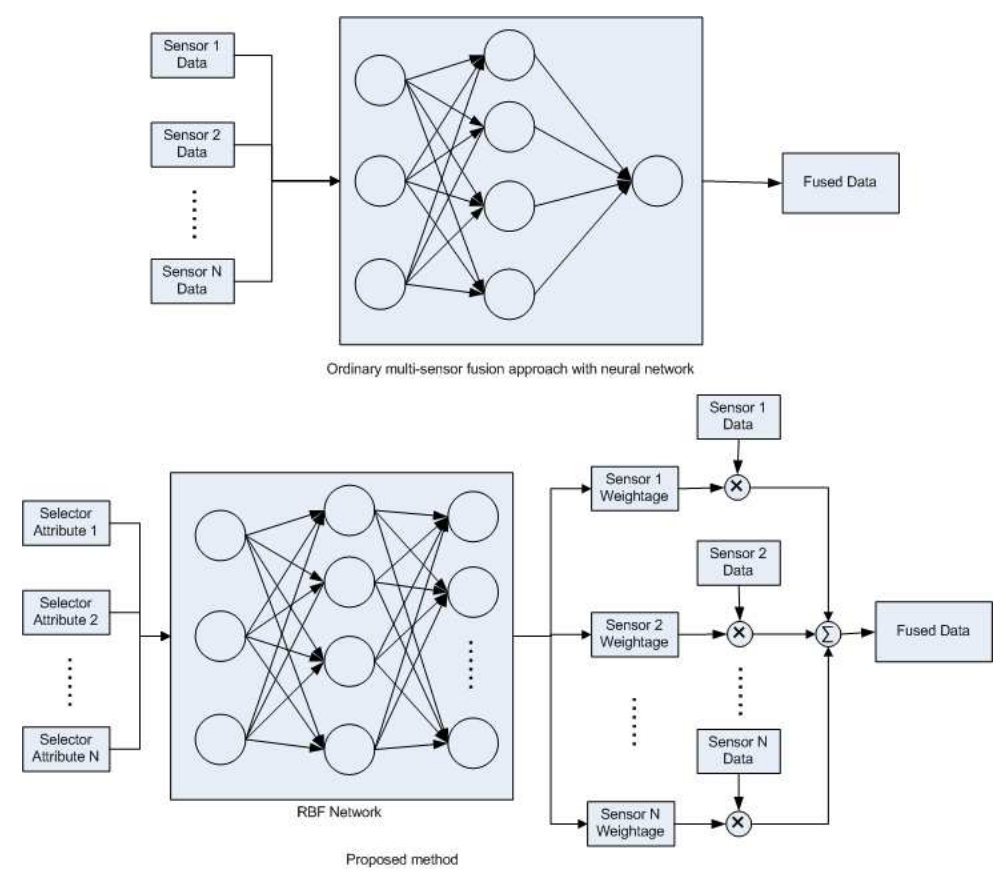

Figure 1: Ordinary multi-sensor fusion approach with neural network and the proposed method

the proper measurement of raw data under different situations such as environmental noise and operational speed. Thus proper monitoring and controlling the system operational and environmental characteristics is also a pre-requirement to conduct the research.

The rest of this paper is organized as follows: Section II describes the proposed RBF neural network based sensor fusion framework based on multiple position sensors and a set of selector attributes. Section III describes the selection and training of the RBF networks. Section IV furnishes the experimental work and results and Section $\mathrm{V}$ concludes this paper.

\section{Proposed Framework}

The main idea of the proposed framework is to facilitate the derivation of a single combined measurement from multiple sensors providing the same type of measurement. Each sensor can be different in the operational principles and thus individually optimal for a restricted set of scenarios classified under a set of selector attributes. The proposed frame work is shown in Fig. 2 and Eq. 1. The objective is to achieve a higher quality measurement in some sense of the application, not from any of 


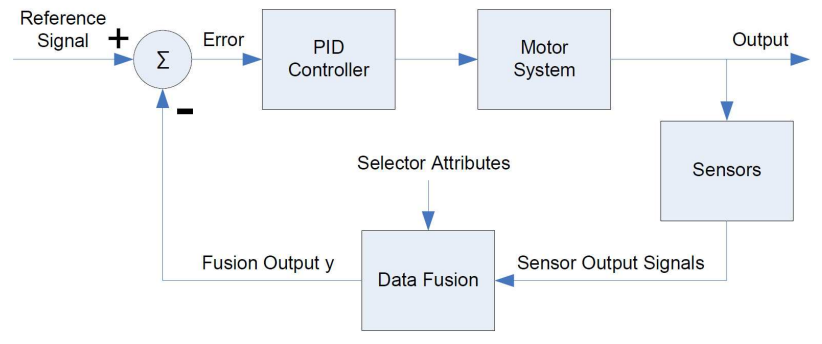

Figure 2: Architecture of the proposed data fusion framework

the individual sensors alone, but an appropriate fusion of the multiple sensors to yield a more optimal fit to the true values in a dynamic manner. While the framework is general, it will be elaborated with respect to position sensing in motion control in this paper and substantiated similarly with a linear motor setup and experiments.

$$
y(s, k)=f\left(s_{1}, s_{2}, \ldots, s_{u}\right) \cdot g\left(k_{1}, k_{2}, \ldots, k_{v}\right)
$$

where $s_{1}, s_{2}, \ldots, s_{u}$ are the sensor output signals of the multiple sensors, $k_{1}, k_{2}, \ldots, k_{v}$ are the selector attributes of the sensor outputs, and $y$ is the data fusion output.

\subsection{Position computation using multiple position sensors}

Assuming that a total number of $\mathrm{N}$ position sensors are used to infer a single position measurement and the fusion function is chosen to be linear with respect to the measurements as shown in Eq. 2 and Fig. 3:

$$
\begin{aligned}
x_{\text {pos }}(k, x) & =\sum_{i=1}^{N} k_{i} x_{\text {snsr }_{i}} \\
& =k_{1} x_{\text {snsr } r_{1}}+k_{2} x_{s n s r_{2}}+\ldots+k_{N} x_{s n s r_{N}}
\end{aligned}
$$

where $k_{1}, k_{2}, \ldots, k_{N}$ are parameters weighing the influence of each of the measurement on the final value, and $x_{s n s r_{1}}, x_{s n s r_{2}}, \ldots, x_{s n s r_{N}}$ represent the measurements from the $N$ different sensors.

We would like to compute the weights so that the target combined value approaches the true value. It should be pointed out that the optimal weights are not fixed but vary with different scenarios which distinguish the strengths of each of the sensor. We refer to the attributes classifying these scenarios as the selector attributes. The true value is unknown

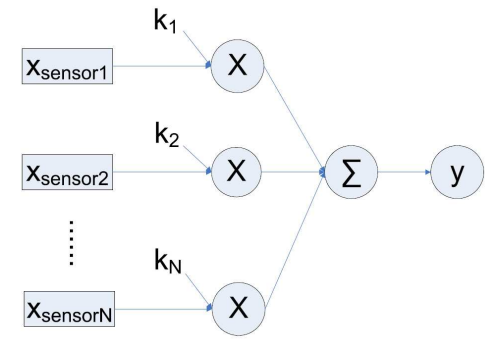

Figure 3: Position computation using multiple position sensors

in practice but it may be taken to be the value of a close calibration from a gold standard calibration sensor.

\subsection{Selection weightage computation}

For a fixed set of selector attributes, a laser interferometer with nanometer resolution is used to yield the reference of the true position $x_{\text {laser }}$ and the parameters $k_{1}, k_{2}, \ldots, k_{N}$ are to be tuned to fit the fused position $x_{\text {pos }}$ to be as close as possible to the reference $x_{\text {laser }}[12]$. Since the function is linear with respect to the parameters, they can be obtained efficiently in a non-iterative manner using least squares estimation algorithms.

However, this single set of weights computed is optimal in the least squares sense with respect to the set of selector attributes. This is fine and sufficient if these attributes are not expected to change during the course of the operations expected of the motion system. When they do change, the weights may not be optimal anymore. To this end, a way to efficiently model and compute the parameters over a range of selector attributes will be necessary. Such selectors may include the frequency of the trajectory, the amount or variance of the noise present relatively among the sensors, the velocity or acceleration of the motion, or the positional zones in the working area. The selectors are necessary to bring out the strengths of individual sensors. For example, different sensors have different characteristics such as response time, bandwidth, resolution, robustness to environmental factors, physical size and cost. A higher weight should be assigned to the specific sensor once the operation enters a domain where it offers relatively higher performance above the others.

In this paper, we consider two selector attributes in the motion velocity and noise level $n_{\text {noise }}$ in each sensor. For a controlled variation in each of these 
attributes, the optimal values of $k_{1}, k_{2}, \ldots, k_{N}$ are computed to correspond to the least square estimation between the computed $x_{\text {pos }}$ and the measured $x_{\text {laser }}$. For the linear data fusion function used in Eq. 2, the equation can be re-written as the following matric-vector form:

$$
\begin{aligned}
x_{\text {pos }} & =S K \\
& =\left[\begin{array}{ccc}
x_{\text {sensor }_{1}}\left(t_{1}\right) & \ldots & x_{\text {sensor }_{N}}\left(t_{1}\right) \\
x_{\text {sensor }_{1}}\left(t_{2}\right) & \ldots & x_{\text {sensor }_{N}}\left(t_{2}\right) \\
\ldots & \ldots & \ldots \\
x_{\text {sensor }_{1}}\left(t_{M}\right) & \ldots & x_{\text {sensor }_{N}}\left(t_{M}\right)
\end{array}\right] \cdot\left[\begin{array}{c}
k_{1} \\
k_{2} \\
\ldots \\
k_{N}
\end{array}\right]
\end{aligned}
$$

where the entries of $S$ are given by $x_{\text {sensor }_{i}}\left(t_{j}\right)$ which is the output of the sensor $i$ at time $t_{j}(i=$ $1,2, \ldots, N ; j=1,2, \ldots, M)$, the entries of $K$ are given by $k_{i}$, and the entries of $x_{\text {pos }}$ are given by $x_{p o s}\left(t_{j}\right)$ at time $t_{j}$.

To find the values of $K$ using the least square estimation between $x_{\text {pos }}$ and $x_{\text {laser }}$, let Eq. 3 equals to $x_{\text {laser }}$ :

$$
S K=x_{\text {laser }}
$$

where the entries of $x_{\text {laser }}$ are given by $x_{\text {laser }}\left(t_{j}\right)$ at time $t_{j}$.

Assuming that $S^{T} S$ is non-singular, the least squares optimal solution of $K$ is given by Eq. 4:

$$
K=\left(S^{T} S\right)^{-1} S^{T} x_{\text {laser }}
$$

where the entries of $x_{\text {laser }}$ are given by $x_{\text {laser }}\left(t_{j}\right)$ at time $t_{j}$.

A recursive least square algorithm can be used to refine the weights with each incremental data set obtained, so that estimation can begin after the first data set and each subsequent incoming data set will improve the accuracy of the estimates. With this algorithm, the optimal values of the weightings $k_{1}, k_{2}, \ldots, k_{N}$ for each set of selector attributes can be obtained to yield a position output closest to the true value under the condition reflected by the selector attributes. To this end, systematic errors such as certain geometrical errors are also compensated in the process. RBF networks, which would be introduced in the next section, are then trained to model those weights over the variation of the attributes.

\section{Parameter Weightage Modeling Using RBF Neural Network}

In this proposed approach, each computed parameter weightage $K$ varies with motion velocity and noise level $n_{\text {noise }}$ in a nonlinear manner. RBF network is used to estimate the weightages in the proposed multi-sensor fusion framework. RBF is a real-valued function where the output only depends on the distance from the origin or some center point $c$ to its input [13]. The following equation describes the RBF:

$$
\varphi(x-c)=\varphi(\|x-c\|)
$$

Derived from function approximation theory, the RBF network is a kind of feed-forward network. They form mappings from an input vector to an output vector. One of the most common RBF is the Gaussian function. Let $y(x)$ be a smooth function from $R$ to $R$. Then, given a compact $S \in R$ and a positive number $w_{M}$, there exists an $\mathrm{RBF}$ network such that with $\left\|w_{0}\right\|<w_{M}$ for all $x \in S$.

$$
y(x)=\sum_{i=1}^{M} w_{i} \varphi_{i}\left(\left\|x-\mu_{i}\right\|\right)+w_{0}
$$

where $M$ is the size of the input vector, $w_{i}$ is the representative value vector, $\varphi_{i}$ is the Gaussian function which $\varphi_{i}\left(\left\|x-\mu_{i}\right\|\right)=\exp \left(-\frac{\left\|x-\mu_{i}\right\|^{2}}{2 \sigma_{i}^{2}}\right), \mu_{i}$ is the basis center of RBF, and $\sigma_{i}$ is the standard deviation.

In order to compute the nonlinear functions associated with the weightages, RBF network should be trained using input and output data sets to obtain the function parameters: $w$ and $\mu$. Here the gradient descent method based on error back propagation is adopted as the training algorithm for parameter adaptation.

Assuming that there is a network with differentiable RBF activation functions, a minimal error can be achieved with the derivatives of back propagated error $E=y_{T}-y_{i}$ vanishing with weights $w$ and basis center $\mu$. Define the parameter set $W=\left(w_{i}, \mu_{i}\right)$, and $\eta$ as the learning rate, then the update of the parameters can be done as:

$$
W(t+1)=W(t)-\eta \nabla E(W(t))
$$

The discrete time version of this algorithm is given as [14]: 


$$
\begin{gathered}
w_{i}(t+1)=w_{i}(t)-\eta_{w} E \varphi_{i}\left(\left\|x-\mu_{i}\right\|^{2}\right) \\
\mu_{i}(t+1)=\mu_{i}(t)-\eta_{\mu} E w_{i} \varphi_{i}\left(\left\|x-\mu_{i}\right\|^{2}\right)\left(x_{T}-\mu_{i}\right) / \sigma_{i}^{2}
\end{gathered}
$$

where $E$ is the back propagated error $y_{T}-y_{i}, y_{T}$ is the target RBF output, $x_{T}$ is the ideal RBF input that yields the desired RBF output, $\eta_{w}$ and $\eta_{\mu}$ are the learning rate of $w$ and $\mu$ and $\sigma_{i}$ is chosen as a constant. A termination condition $E_{m s}$, the mean square of errors, is adopted here to end the iterative adaption process and obtain the optimum RBF parameters.

In the case study considered in this paper to be further highlighted in Section IV, the two selector attributes in terms of the velocity velo and noise level $n_{\text {noise }}$ in the velocity sensor are considered as the input $x$. The selection weightage $K$ computed using least squares estimation is used as the target $y$. Thus, this specific RBF network has 2-DOF (degree-of-freedom) since its characteristics depend on both motion velocity velo and noise level $n_{\text {noise }}$. Based on Eq. 7, this RBF network can be re-written as:

$K=\sum_{i=1}^{M} w_{i} \varphi_{i}\left(\|\left[\right.\right.$ velo, $\left.\left.n_{\text {noise }}\right]-\left[\mu_{\text {velo }_{i}}, \mu_{n_{\text {noise }} i}\right] \|\right)+w_{0}$

The RBF weights $w$ can be obtained by replacing $x$ and $\mu$ with $\left[\right.$ velo, $\left.n_{\text {noise }}\right]$ and $\left[\mu_{\text {velo }}, \mu_{n_{\text {noise }}}\right]$ accordingly in Eq. 9 and Eq. 10. The trained RBF function can be used to model the variation of the weightages $K$ with the selector attributes and subsequently used to construct the data fusion function accordingly using Eq. 3 .

\section{Case Study}

A single axis setup (Akribis DC linear motor) is used as the basis for a case study. The slide attached on the stage can move bi-directionally along the $\mathrm{X}$-axis, with a working range of $200 \mathrm{~mm}$. A magnetic encoder with a resolution of $2 \mathrm{~mm}$ is used to obtain the position signal $x_{e n c}$. An analog velocity sensor is also mounted to give analog velocity measurements as well as positional information when the velocity is fed into a digital integrator. The two sensors work independently and no communication is required between them. Thus, in this case study, we have two different sources of position measurement; one from the magnetic encoder

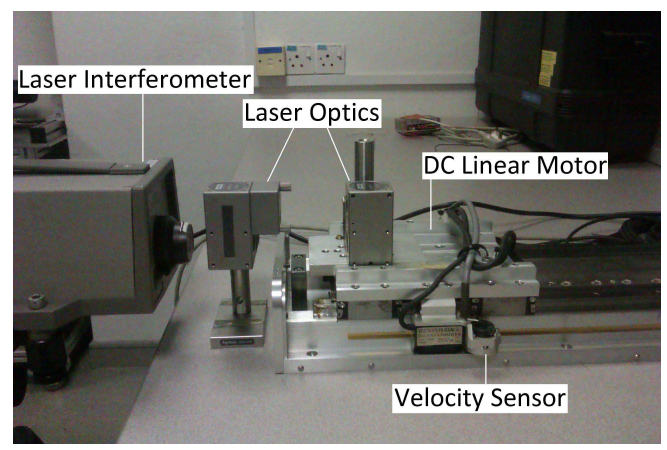

Figure 4: System setup 1

and the other from the velocity sensor integrated to yield the position. Being an analog sensor, the resolution of the velocity sensor is infinitesimal. Thus, when digital integration is done accurately over the lower velocity range, the latter position measurement can offer a better accuracy than the magnetic encoder. However, as velocity picks up relative to the sensitivity of the sensor, the accuracy of the velocity measurement degrades leading to accuracy loss in the position measurements too after the digital integration. Thus, the relative performance of each of the sensor varies with velocity and velocity is selected as the first selector attribute in this case study. The relative amount of noise present from the two sources should also govern the priority to be attached to each of the two measurements to infer the final position measurement. Thus, the second selector attribute is chosen to be the amount of noise present in the velocity measurements.

A laser interferometer is used to provide the "true" position measurement $x_{\text {laser }}$. This laser interferometer is only used in the selection weightage computation stage and is not used in the system operation. The system setup is shown in Fig. 4 and Fig. 5.

\subsection{Data collection phase}

The motor is controlled to run at constant velocity mode with $200 \mathrm{~mm}$ total movement length. The noise level associated with the velocity sensor used can be maintained at $5 \%$ in ideal and static laboratory conditions. However, it can vary with the presence and interaction with EM sources, power interference or operating conditions. To enable the results to remain applicable at other times and operating conditions of the motor, the noise level as a selector attribute is allowed to vary during the data collection phase by injecting or amplifying the noise 


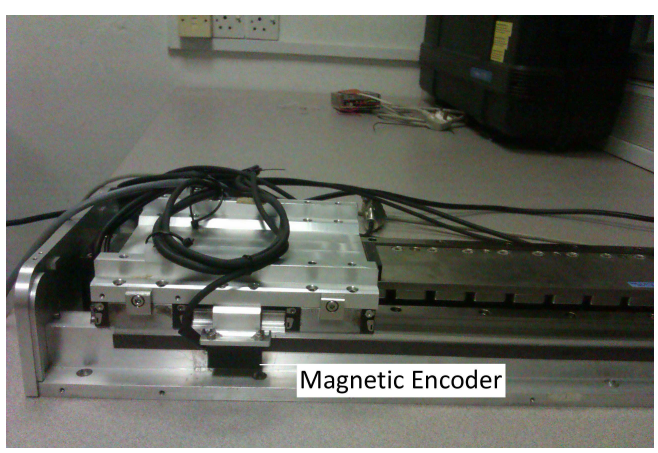

Figure 5: System setup 2

to different levels. The raw data and true data are collected with different velocities from $0.02 \mathrm{~m} / \mathrm{s}$ to $0.6 \mathrm{~m} / \mathrm{s}$ and different noise levels in the velocity sensor from $5 \%$ to $500 \%$ of the maximum reading for each velocity. At a specific movement velocity and a specific noise level in the velocity sensor, the velocity sensor output $v_{v s e n s o r}$, the magnetic encoder output $x_{e n c}$ and the true data $x_{l a s e r}$ are continuously collected during the motor movement, and the velocity sensor measured position signal $x_{v s e n s o r}$ is obtained separately after the integration of $v_{v s e n s o r}$.

\subsection{Parameter estimation}

Using the linear in the parameter form, the combined position can be expressed in the following form:

$$
x_{\text {pos }}=k_{1} x_{v s e n s o r}+k_{2} x_{\text {enc }}
$$

The reference position $x_{\text {laser }}$ obtained by the laser interferometer is used as the reference position $x_{\text {laser }}$ for the purpose of estimating the parameters $k_{1}$ and $k_{2}$. For each set of selector attributes, the optimal weight parameters can thus be estimated with the algorithm as presented in previous section. Thus, with the full data set collected earlier, two tables (Table 1 and 2) can be obtained, tabulating the optimal weights against the velocity selector $v_{v s e n s o r}$ and the noise selector $n_{\text {noise }}$.

\subsection{RBF modeling of weights variation}

In this specific case, a 2-dimensional RBF networks is trained and applied to approximate the values of the weight parameters $k_{1}$ and $k_{2}$ as shown in Fig. 6 based on two selector inputs: $v_{v s e n s o r}$ and $n_{\text {noise }}$ as follows:

$$
k_{1}=f_{k_{1}}\left(v_{\text {vsensor }}, n_{\text {noise }}\right)
$$

Table 1: $k_{1}$ Selection for Different Velocities and Noise Levels

\begin{tabular}{llllllll}
\hline \multirow{2}{*}{ Noise } & \multicolumn{7}{c}{ Velocities $(\mathrm{m} / \mathrm{s})$} \\
\cline { 2 - 8 } & 0.04 & 0.10 & 0.20 & 0.30 & 0.40 & 0.50 & 0.60 \\
\hline $5 \%$ & 0.87 & 0.875 & 0.61 & 0.46 & 0.25 & 0.29 & 0.00 \\
\hline $10 \%$ & 0.87 & 0.885 & 0.62 & 0.47 & 0.255 & 0.29 & 0.00 \\
\hline $50 \%$ & 0.89 & 0.98 & 0.585 & 0.405 & 0.30 & 0.265 & 0.00 \\
\hline $100 \%$ & 0.915 & 1.00 & 0.665 & 0.48 & 0.185 & 0.31 & 0.00 \\
\hline $300 \%$ & 0.825 & 1.00 & 0.48 & 0.285 & 0.025 & 0.105 & 0.00 \\
\hline $500 \%$ & 0.375 & 0.38 & 0.205 & 0.01 & 0.005 & 0.055 & 0.00 \\
\hline
\end{tabular}

Table 2: $k_{2}$ Selection for Different Velocities and Noise Levels

\begin{tabular}{llllllll}
\hline \multirow{2}{*}{ Noise } & \multicolumn{7}{c}{ Velocities $(\mathrm{m} / \mathrm{s})$} \\
\cline { 2 - 8 } & 0.04 & 0.10 & 0.20 & 0.30 & 0.40 & 0.50 & 0.60 \\
\hline $5 \%$ & 0.135 & 0.13 & 0.39 & 0.54 & 0.75 & 0.705 & 0.995 \\
\hline $10 \%$ & 0.135 & 0.12 & 0.38 & 0.53 & 0.745 & 0.705 & 0.995 \\
\hline $50 \%$ & 0.115 & 0.025 & 0.42 & 0.6 & 0.7 & 0.73 & 0.995 \\
\hline $100 \%$ & 0.09 & 0.01 & 0.34 & 0.525 & 0.82 & 0.69 & 0.995 \\
\hline $300 \%$ & 0.185 & 0.035 & 0.54 & 0.73 & 0.98 & 0.895 & 0.995 \\
\hline $500 \%$ & 0.65 & 0.665 & 0.82 & 1.00 & 1.00 & 0.945 & 0.995 \\
\hline
\end{tabular}

$$
k_{2}=f_{k_{2}}\left(v_{\text {vsensor }}, n_{\text {noise }}\right)
$$

The fused position signal can be represented as in Eq. 15, and this position signal $x_{\text {pos }}$ can either be used directly by the controller, or be interpolated to a higher order to improve the system resolution.

$$
\begin{aligned}
x_{\text {pos }}= & f_{k_{1}}\left(v_{\text {vsensor }}, n_{\text {noise }}\right) x_{\text {vsensor }} \\
& +f_{k_{2}}\left(v_{\text {vsensor }}, n_{\text {noise }}\right) x_{\text {enc }}
\end{aligned}
$$

The approximated functions $f_{k_{1}}\left(v_{v s e n s o r}, n_{\text {noise }}\right)$ of $k_{1}$ and $f_{k_{2}}\left(v_{v s e n s o r}, n_{\text {noise }}\right)$ of $k_{2}$ are determined and depicted in Fig. 7 and Fig. 8, under different velocities and noise levels. A nonlinear relationship can be observed between $k_{1} / k_{2}$ values and selectors: velocity and noise levels. $k_{1}$ decreases significantly with higher noise levels under same velocity value. This is consistent with physical intuition as a higher content of noise will shift weightage away from the analog sensor. $k_{1}$ also decreases significantly with higher velocity under same noise level. At lower velocities, the position measurements from the digitally integrated velocity signal yield good accuracy

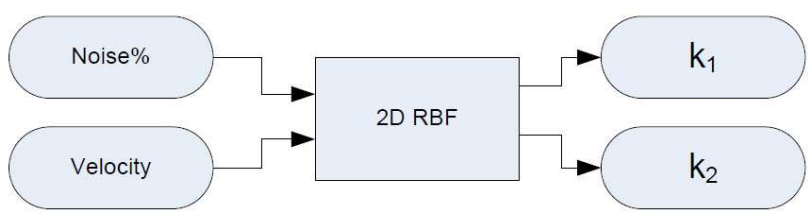

Figure 6: Flowchart of 2D RBF network 


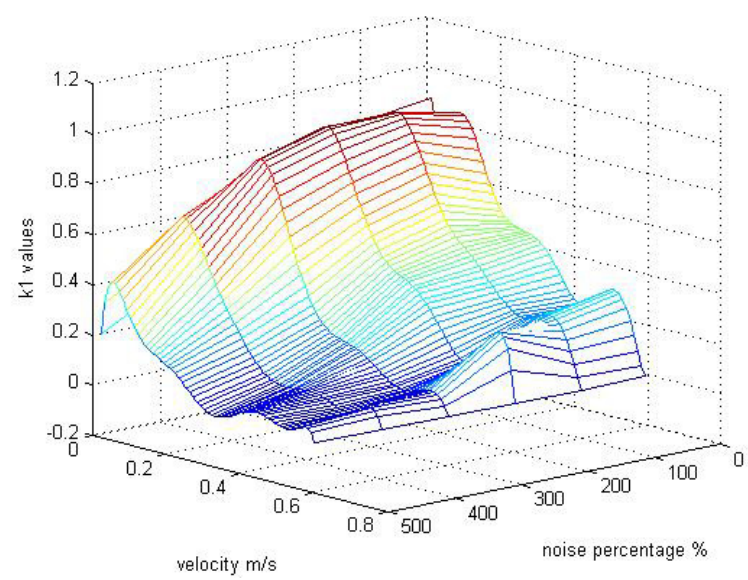

Figure 7: RBF approximation of $k_{1}$

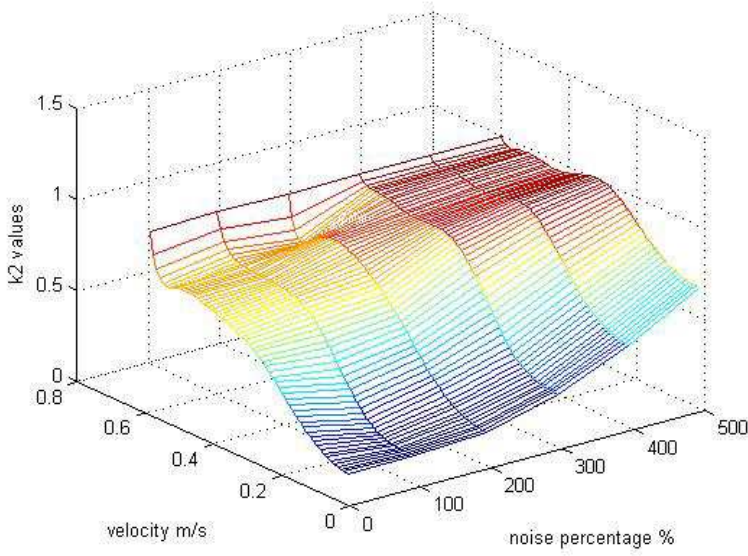

Figure 8: RBF approximation of $k_{2}$

but the accuracy is degraded at higher velocities due to the sensitivity characteristics of the sensor. The complementary relationships are observed in $k_{2}$.

\subsection{Results \& Discussions}

To observe the improvement on the overall precision with the proposed framework and approach, control experiments on the testbed are done. Three sinusoidal reference signals with the amplitude of $100 \mathrm{~mm}$ and the periods of $3 / 4 / 5$ seconds are applied to the linear motor separately. The periods of the sinusoidal reference signals are specially chosen to include all the measured velocities of the linear machine. A PID controller is used to control the linear motor. The analog velocity sensor is used to measure the velocity signal $v_{v s e n s o r}$, the magnetic encoder is used to measure the position signal $x_{e n c}$ and the laser interferometer is used to measure the reference position $x_{\text {laser }}$. Those collected signals can be used to train the 2-dimensional RBF networks, and the estimated position $x_{p o s}$ can be obtained using Eq.15. The positioning and tracking performances of the proposed method for the three reference sinusoidal signals are shown in Fig. 9 to Fig. 11.

To observe the error tolerance of the proposed approach, the system tracking performance at different noise levels are plotted from Fig. 12 to Fig. 17, for reference signals with period of 5 seconds and error percentages of $5 \%, 10 \%, 20 \%, 30 \%, 40 \%$ and $50 \%$. As can be clearly observed in the experimental study, the proposed method can improve the tracking performance and system precision if the noise level is kept below $30 \%$.

In order to verify the efficacy of the proposed method comparing with the ordinary approach from literature review, a RBF network is designed where the measurements from multi-sensors are directly used as the inputs to train the network as the standard neural network shown in Fig. 1. Two sinusoidal reference signals with the amplitude of $100 \mathrm{~mm}$ and the periods of 1 and 2 seconds are applied to the linear motor separately and the estimated position signals from both proposed method and ordinary approach are shown in Fig. 18, together with the reference signals. It can be observed that the estimated position signals from the proposed method are much better than those from the ordinary approach.

The promising results show that in precision motion system the fusion of magnetic encoder and analog velocity sensor using proposed method can provide better results than ordinary approach and individual sensor alone. Although in practice, magnetic encoder has lower resolution than optical encoder, but with lower cost it is less sensitive to vibration and impact and more robust to the presence of environmental contaminants. Therefore in this specific example, magnetic encoder can outperform with respect to some of these requirements using proposed method.

In practice, a digital encoder can be used to yield position measurements with satisfactory accuracy over a good range of the control motion, but a higher accuracy is needed near the target position. Using an additional position sensor in parallel, such as an analog encoder which can be invoked around 


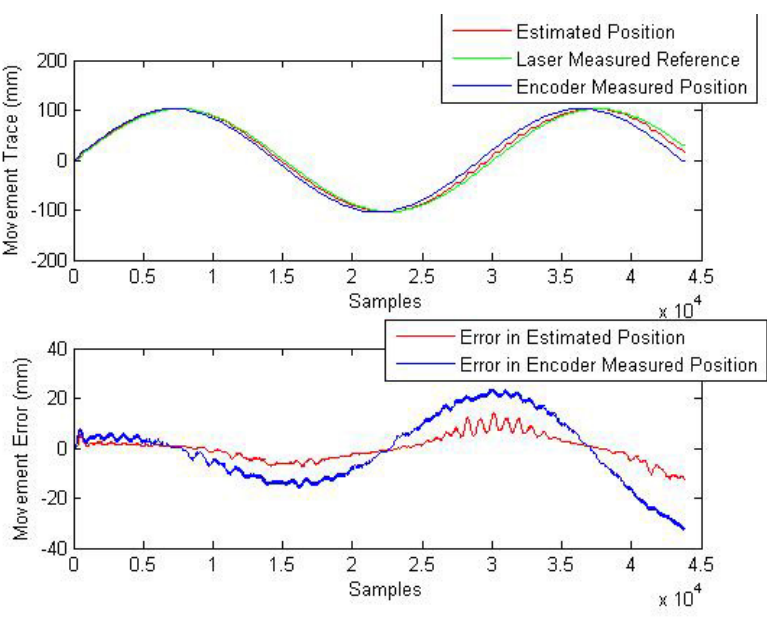

Figure 9: Tracking performance with sinusoidal reference input signal $(\mathrm{amp}=100 \mathrm{~mm}$ period $=3 \mathrm{~s})$
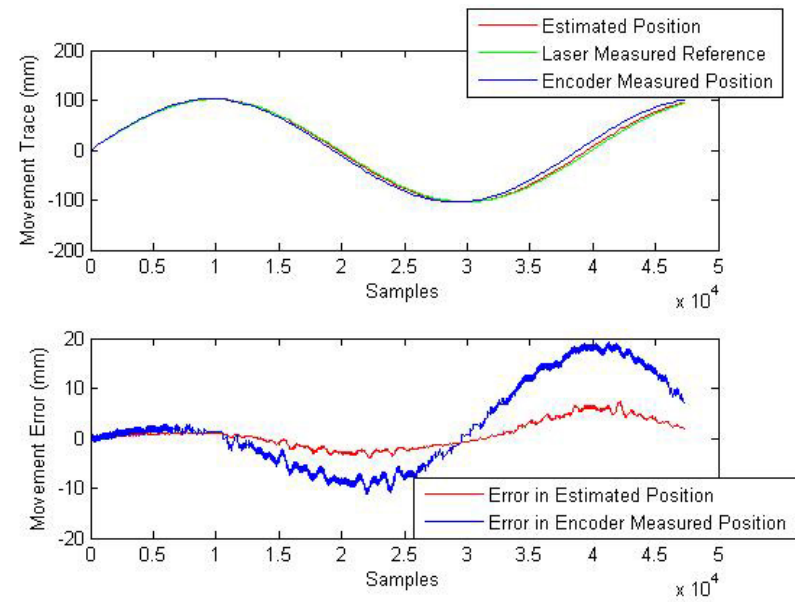

Figure 10: Tracking performance with sinusoidal reference input signal $(\mathrm{amp}=100 \mathrm{~mm}$ period $=4 \mathrm{~s})$

the settling point to yield or interpolate into finer resolution measurements [15][16], can fill the gaps beyond the reach of the other sensor. The potential of the use of multiple different sensors for the same measurement is shown, each suited to a certain set of operational factors possibly and collectively arising in the same application. Other applications may include an appropriate synergy of position sensors to achieve dynamic balance in response speed and resolution, bandwidth and accuracy, cost and performance, robustness and sensitivity, or other combination of these attributes.
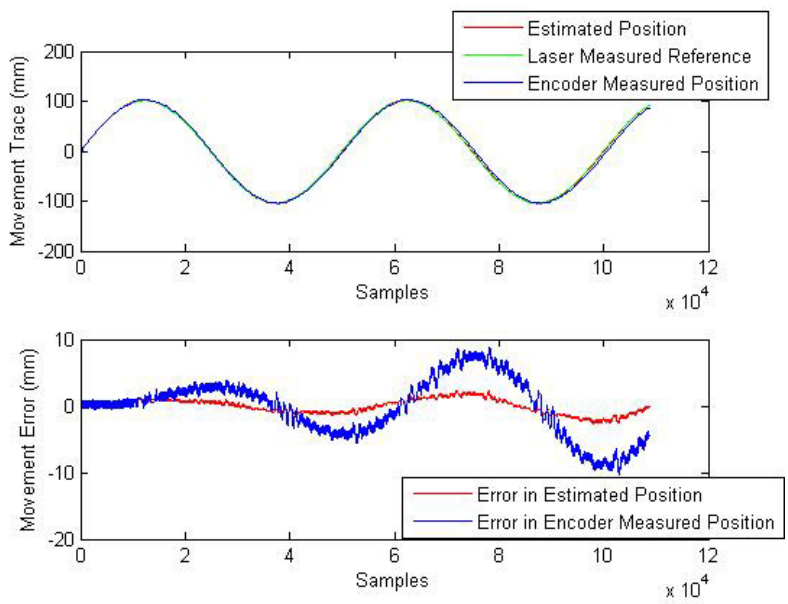

Figure 11: Tracking performance with sinusoidal reference input signal $(\mathrm{amp}=100 \mathrm{~mm}$ period $=5 \mathrm{~s})$
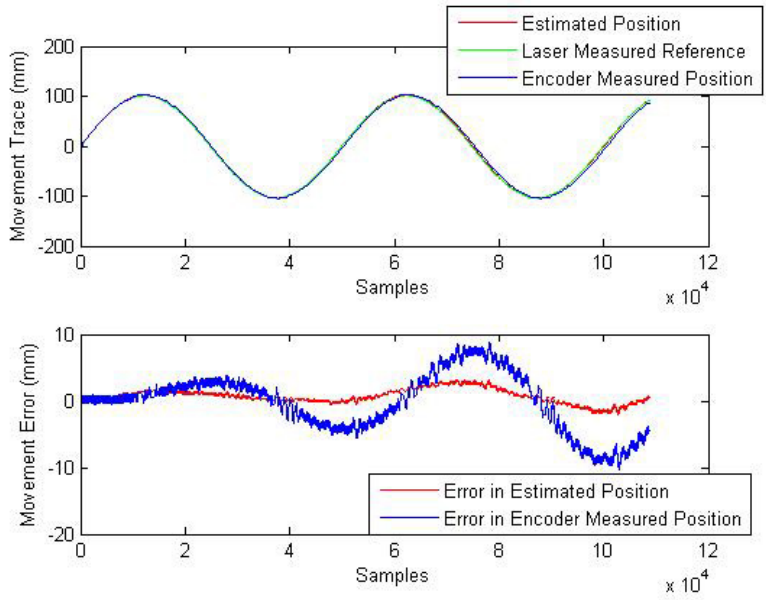

Figure 12: Tracking performance with $5 \%$ velocity sensor error and sinusoidal reference input signal $(\mathrm{amp}=100 \mathrm{~mm}$ pe$\operatorname{riod}=5 \mathrm{~s}$ ) 

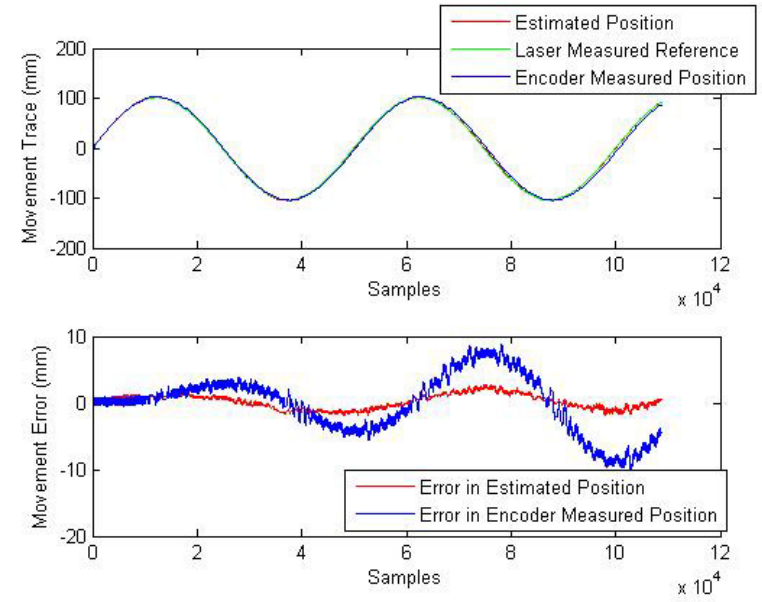

Figure 13: Tracking performance with $10 \%$ velocity sensor error and sinusoidal reference input signal $(\mathrm{amp}=100 \mathrm{~mm}$ pe$\operatorname{riod}=5 \mathrm{~s})$
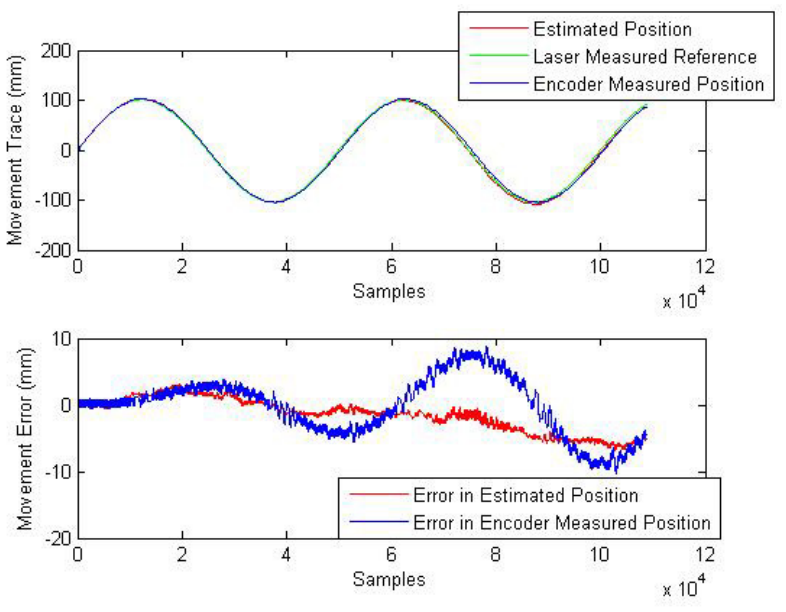

Figure 14: Tracking performance with $20 \%$ velocity sensor error and sinusoidal reference input signal $(\mathrm{amp}=100 \mathrm{~mm}$ pe$\operatorname{riod}=5 \mathrm{~s})$
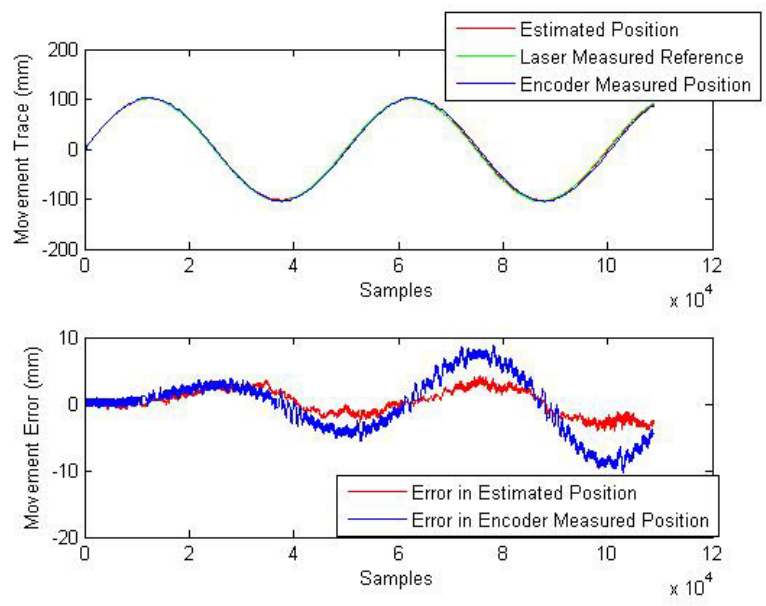

Figure 15: Tracking performance with $30 \%$ velocity sensor error and sinusoidal reference input signal $(\mathrm{amp}=100 \mathrm{~mm}$ pe$\operatorname{riod}=5 \mathrm{~s}$ )
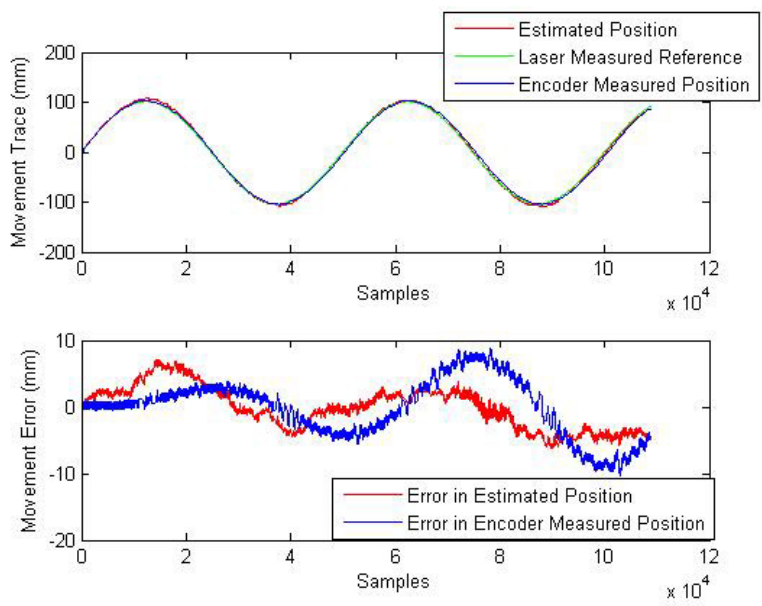

Figure 16: Tracking performance with $40 \%$ velocity sensor error and sinusoidal reference input signal $(\mathrm{amp}=100 \mathrm{~mm}$ pe$\operatorname{riod}=5 \mathrm{~s})$ 

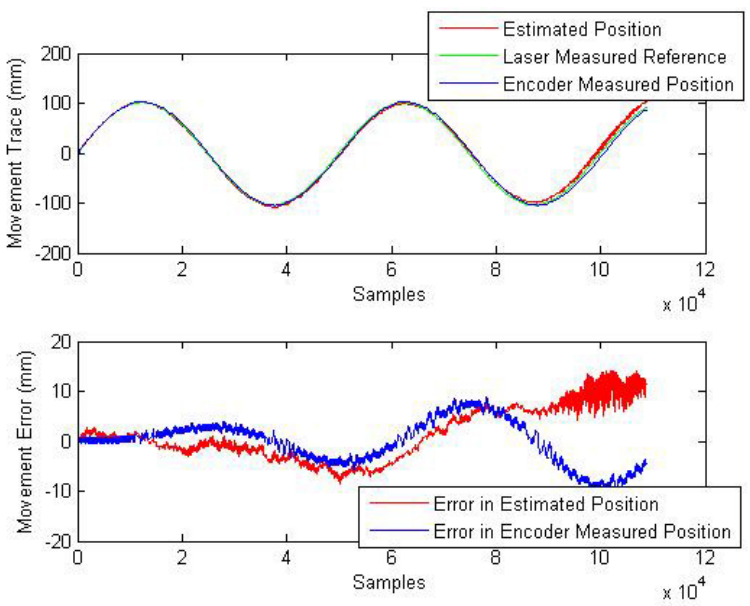

Figure 17: Tracking performance with $50 \%$ velocity sensor error and sinusoidal reference input signal $(\mathrm{amp}=100 \mathrm{~mm}$ pe$\operatorname{riod}=5 \mathrm{~s}$ )

\section{Conclusion}

A RBF neural network based framework to fuse measurements from different sensors in a practically amenable manner has been presented in this paper. The weight attached to each measurement is not fixed but evolves with selector attributes to yield an improved combined measurement based on the situation as demarcated by the attributes. A case study on a linear motor with two position measurements forthcoming from two different sensors has been demonstrated and improved performance has been observed compared with the approach with a single sensor and the ordinary neural network based multi-sensor approach.

\section{References}

[1] J. N. Gross, Y. Gu, M. B. Rhudy, S. Gururajan, M. R. Napolitano, Flight-test evaluation of sensor fusion algorithms for attitude estimation, Aerospace and Electronic Systems, IEEE Transactions on 48 (3) (2012) 2128-2139.

[2] V. Chan, C. Bradley, G. Vickers, A multi-sensor approach to automating co-ordinate measuring machinebased reverse engineering, Computers in Industry 44 (2) (2001) 105-115.

[3] T.-S. Shen, J. Huang, C.-H. Menq, Multiple-sensor integration for rapid and high-precision coordinate metrology, Mechatronics, IEEE/ASME Transactions on 5 (2) (2000) 110-121.

[4] Y. Luo, T. Guan, B. Wei, H. Pan, J. Yu, Fast terrain mapping from low altitude digital imagery, Neurocomputing 156 (2015) 105-116.

[5] S. Adusumilli, D. Bhatt, H. Wang, V. Devabhaktuni, P. Bhattacharya, A novel hybrid approach utilizing principal component regression and random forest regression to bridge the period of gps outages, Neurocomputing.

[6] D. Amarasinghe, G. K. Mann, R. G. Gosine, Landmark detection and localization for mobile robot applications: A multisensor approach, Robotica 28 (05) (2010) 663673.

[7] B. Siciliano, O. Khatib, Springer handbook of robotics, Springer Science \& Business Media, 2008.

[8] S. S. Chiddarwar, N. R. Babu, Comparison of rbf and mlp neural networks to solve inverse kinematic problem for $6 \mathrm{r}$ serial robot by a fusion approach, Engineering applications of artificial intelligence 23 (7) (2010) 10831092.

[9] N. Ghosh, Y. Ravi, A. Patra, S. Mukhopadhyay, S. Paul, A. Mohanty, A. Chattopadhyay, Estimation of tool wear during cnc milling using neural network-based sensor fusion, Mechanical Systems and Signal Processing 21 (1) (2007) 466-479.

[10] B. Sick, On-line and indirect tool wear monitoring in turning with artificial neural networks: a review of more than a decade of research, Mechanical Systems and Signal Processing 16 (4) (2002) 487-546. 
[11] R. C. Luo, C.-C. Yih, K. L. Su, Multisensor fusion and integration: approaches, applications, and future research directions, Sensors Journal, IEEE 2 (2) (2002) $107-119$.

[12] R. Yang, J. Fuh, J. Sun, A. Tay, K. K. Tan, Displacement and thermal error compensation using rbf networks, Control and Intelligent Systems 42 (4).

[13] I. Aizenberg, Complex-valued neural networks with multi-valued neurons, Vol. 353, Springer, 2011.

[14] C. M. Bishop, Neural networks for pattern recognition, Oxford university press, 1995.

[15] K. K. Tan, H. X. Zhou, T. H. Lee, New interpolation method for quadrature encoder signals, Instrumentation and Measurement, IEEE Transactions on 51 (5) (2002) 1073-1079.

[16] K. K. Tan, K.-Z. Tang, Adaptive online correction and interpolation of quadrature encoder signals using radial basis functions, Control Systems Technology, IEEE Transactions on 13 (3) (2005) 370-377. 